\title{
Computer Control the Accuracy of the Parts Processed on CNC Machine Tools Based on Statistical Process Control
}

\author{
Angel Lengerov, Galina Nikolcheva², Julieta Kaleycheva², Lyubomir Lazov ${ }^{3}$ \\ ${ }^{I}$ Department of Machine Tools and Mechanical Engineering, Technical University, Plovdiv, Bulgaria, \\ ${ }^{2}$ Department of Machine Tools and Mechanical Engineering, Technical University, Sofia, Bulgaria, ${ }^{3}$ Rezekne \\ Academy of Technologies, Latvia
}

\begin{abstract}
Statistical process control (SPC) can help manufacturers improve the quality of their products. This paper describes a relatively simple and effective method and algorithm to adjust the accuracy of parts produced on CNC machine tools using the methods of statistical process control. Examined the possibilities of the proposed method by computer simulation of the process. By using the proposed algorithm scattering of size is placed in the middle of the tolerance zone. Various variants carrying out the method are proposed adequate on the machining time during processing details in the sample. This can optimize the total time for processing of the batch.
\end{abstract}

Keywords: machining, CNC machine tools, computer control, statistical control.

\section{INTRODUCTION}

$\mathrm{CNC}$ machine tools $\mathrm{c}$ used in modern engineering production has increased radically over the last few decades. Manufacturing requirements along with quality requirements have become progressively more stringent for achieving high-quality products [3]. One way to improve accuracy in the production of machines parts is to use methods of statistical process control. These methods allow to apply in process control systems. Statistical Process Control (SPC) has traditionally been used in manufacturing systems to analyses and interpret measurement results and to provide compensation parameters [8]. SPC can help manufacturers improve the quality of their products. It is effective in controlling the fluctuations of the manufacturing process. SPC utilizes statistical methods to monitor manufacturing processes with an aim to maintain and improve the product quality while decreasing the variance [4].

Many researchers as Falsone [5], Nani [6] and Ramamoorthy and Radhakrishnan [7], develop and implement schemes for statistical control of production quality improvement and productivity. SPC helps define the capability of the stable process to judge whether it is operating at an acceptable. Although in modern information technology is accumulated a huge amount of databases from prior production experience, any new device is required to provide initial information for carrying out the process and control the quality. Automated data collection, low cost calculation, and demands for higher quality, lower cost, and greater reliability are the cause of using SPC [4]. The statistical analysis of the measurement results provides information about the technological process stability [6]. The aim of this article is to create a method and algorithm to adjust the accuracy of parts produced on CNC machine tools using the methods of statistical process control.

\section{METHODOLOGY}

In the process of making a series of identical parts are controlled the dimensional accuracy, the location of the surfaces and material properties. These are indicators for the accuracy of the products. For each is given norms of accuracy and limits deviations. These characteristics are controlled by their statistical evaluations [1].

Before the processing of each batch is carried dimensional setting. This is done in order to get as close to the working adjusting size $A_{u p}$. Where the rate of tool wear has a great influence, the size is determined by the expression:

$$
A_{н p}=A+E M \mp\left(0,5 T-\omega_{u з м}-0,5 \omega\right),
$$

where: $A$ is the nominal size of surface; $T$ is the tolerance size; $E M$ is the average deviation of the tolerance zone; $\omega_{\text {изм }}$ is error of the measurement at setup; $\omega$ is field of scattering. The "-"refers to the processing of shafts; the sign "+" refers to the processing of holes.

From equation (1) that to determine $A_{\mu p}$ need to know the field of scattering of the upcoming manufacturing process. Unlike mass and large series

ISSN 1691-5402

(C) Rezekne Academy of Technologies, Rezekne 2017 http://dx.doi.org/10.17770/etr2017vol3.2623 
production in small batch production and small amount of batch thus information is not available. For determination of $A_{t p}$, apply different solutions.

Classical approach is the use of small sample test details. The method of small sample apply to processes that are scheduled rarer setups. It successfully applied in large series and mass production. Then the statistical characteristics of the process are known, including the dispersion caused by random factors. It is used primarily to assess the accuracy of the initial static dimensional setting-up.

When using CNC machine tools, the method of the small samples has a different implementation [9]. Carry out initial dimensional setting trial chips to size $A_{u p}^{\prime}=A+E M$. The scattering of the size at the finish machining is considerably less than the tolerance size, so the initial setting has to ensure the production of workable parts. But the initial setting is not optimal in terms of the process to the first settingup. The test sample contains a small number of parts sufficient to an indicative evaluate the dispersions and the working adjusting size. After making the adjustment to the initial setting are controlled the size of each of the following processed details and the accumulating information with appropriate statistical analysis allows for more precise dimensional setting. A different approach for dimensional setting used in [2]. The initial setting is static. Each workpiece is measured and is corrected the dimensional size to stabilize the process. For dimensional control is applied original method by touching the tool with the workpiece.

The experience accumulated for initial dimensional setting warrants the conclusion that at CNC machine tools is appropriate after the initial static setting to exercise ongoing control of all processed parts. The obtained information is processed at real-time statistically. As a result of the analysis is precise dimensional adjustment.

For the realization of this idea was developed following algorithm that has a simple software implementation of the discussed above solutions.

The algorithm is given below.

- Step 1. Static or trial chips is carried out initial dimensional setting of average size:

$$
A_{u p}=A+E M
$$

- Step 2. Starts batch processing, as measured after processing every detail and fills a database of information: $A_{1} ; A_{2} ; A_{3} \ldots$;

- Step 3. After processing the small sample with size $\mathrm{n}$ (at least pieces) started statistical information processing in parallel with the implementation of the manufacturing process.

Calculate at real-time the average sample size and corrections of the dimensional setting to maintain the size-adjusting $A_{\mu p}=A+E M=$ const :

After the first $n$ details:

$$
\bar{A}_{1}=\frac{1}{n} \sum_{i=1}^{n} A_{i} ; \quad \Delta_{H, 1}=A_{\mu p}-\bar{A}_{1} ;
$$

- Step 4. If $\left|\Delta_{H, 1}\right|>0,1 T$,

Then made a correction on to the initial setting with $\Delta A_{\mu p}=\Delta_{H, 1}$

Otherwise, the process continues without correction.

Processing new sample with size $n$ the workpiece, and are carried out the same actions as described above.

Whatever is the process, at the implementation of the proposed algorithm is achieved scattering of the sizes of processed details around the average size. It is the most favorable location at the accuracy diagram in the tolerance zone.

To study the effect of the proposed algorithm is carried out computer simulation of processes with and without dominant systematic factor. The factors which influence on the accuracy of machining of parts are tool wear, thermal deformation, changing the cutting conditions and setting the technological system.

\section{RESULTS AND DISCUSSION}

Table 1 shows a part of the numerical results obtained from the testing of the algorithm with dominant systematic factor at turning of a shaft with size $D=35_{-0,04}$.

$$
\left(A_{u p}=34,98 \mathrm{~mm} ; \quad 0,1 T=0,004 \mathrm{~mm}\right)
$$

\begin{tabular}{|c|c|c|c|c|}
\hline \multicolumn{5}{|c|}{ Table 1} \\
\hline № & $\mathrm{A}$ & $\mathrm{A}+\Delta_{\text {н }}$ & $\mathrm{A}_{\text {cp }}$ & $\Delta_{\text {н }}$ \\
\hline 1 & 34,998 & 34,998 & & \\
\hline 2 & 35,001 & 35,001 & & \\
\hline 3 & 35,006 & 35,006 & & \\
\hline 4 & 34,998 & 34,998 & & \\
\hline 5 & 34,997 & 34,997 & 35 & $-0,02$ \\
\hline 6 & 35,008 & 34,988 & & \\
\hline 7 & 34,991 & 34,971 & & \\
\hline 8 & 35,005 & 34,985 & & \\
\hline 9 & 35,002 & 34,982 & & \\
\hline 10 & 34,99 & 34,970 & 34,979 & 0,001 \\
\hline 11 & 35,006 & 34,986 & & \\
\hline 12 & 34,99 & 34,972 & & \\
\hline 13 & 35,007 & 34,987 & & \\
\hline 14 & 34,997 & 34,977 & & \\
\hline 15 & 34,998 & 34,978 & 34,98 & 0,000 \\
\hline 16 & 35,002 & 34,982 & & \\
\hline
\end{tabular}

In figure 1 shows the diagrams of the process without dominant systematic factor. Above located diagram is computer-generated Gaussian distribution with standard deviation $\sigma=3,5 \mu \mathrm{m}$ compared to the nominal size $\mathrm{D}=35 \mathrm{~mm}$. The second diagram located 
Environment. Technology. Resources, Rezekne, Latvia Proceedings of the $11^{\text {th }}$ International Scientific and Practical Conference. Volume III, 179-182

below the first one presents the results after applying the algorithm for dimensional setup.

Table 2

\begin{tabular}{|c|c|c|c|c|c|}
\hline № & $\mathrm{A}$ & $\mathrm{A}+\Delta_{\mathrm{H}}$ & $\mathrm{A}_{\mathrm{cp}}$ & $\Delta_{\mathrm{H}}$ & $\Sigma \Delta_{\mathrm{H}}$ \\
\hline 1 & 34,998 & 34,998 & & & \\
\hline 2 & 35,003 & 35,003 & & & \\
\hline 3 & 35,01 & 35,01 & & & \\
\hline 4 & 35,004 & 35,004 & & & \\
\hline 5 & 35,005 & 35,005 & 35,004 & $-0,024$ & $-0,024$ \\
\hline 6 & 35,018 & 34,994 & & & \\
\hline 7 & 35,003 & 34,979 & & & \\
\hline 8 & 35,019 & 34,995 & & & \\
\hline 9 & 35,018 & 34,994 & & & \\
\hline 10 & 35,008 & 34,984 & 34,989 & $-0,009$ & $-0,033$ \\
\hline 11 & 35,026 & 34,992 & & & \\
\hline 12 & 35,014 & 34,981 & & & \\
\hline 13 & 35,031 & 34,998 & & & \\
\hline 14 & 35,023 & 34,99 & & & \\
\hline 15 & 35,026 & 34,993 & 34,991 & $-0,011$ & $-0,044$ \\
\hline 16 & 35,032 & 34,988 & & & \\
\hline
\end{tabular}

Table 2 shows a part of the numerical results obtained from the testing of the algorithm with dominant systematic factor at turning of a shaft with size $D=35_{-0,04}$.

$$
\text { ( } A_{\text {up }}=34,98 \mathrm{~mm} ; 0,1 T=0,004 \mathrm{~mm} \text { ) }
$$

In figure 2 shows the diagrams of the process with dominant systematic factor. Above located diagram is computer-generated Gaussian distribution with standard deviation $\sigma=3,5 \mu \mathrm{m}$ compared to the nominal size $D=35 \mathrm{~mm}$. The second diagram located below the first one presents the results after applying the algorithm for dimensional setup.

The figures 1 and 2 shows that and when there is a systematically increase in the dimensions of the machined parts caused by the tool wear, the algorithm successfully maintains the dimensional setting on the average value of size. Moreover, the accuracy of the process is increased considerably. For the specific example, at tendency to increase in size up to 0,002 $\mathrm{mm} / \mathrm{pcs}$ and field of scattering around the center line $\omega=23 \mu \mathrm{m}$; without applying of the algorithm the size scatter is $\omega=100 \mu \mathrm{m}$. When used the algorithm, the size scatter is $\omega=26 \mu \mathrm{m}$.

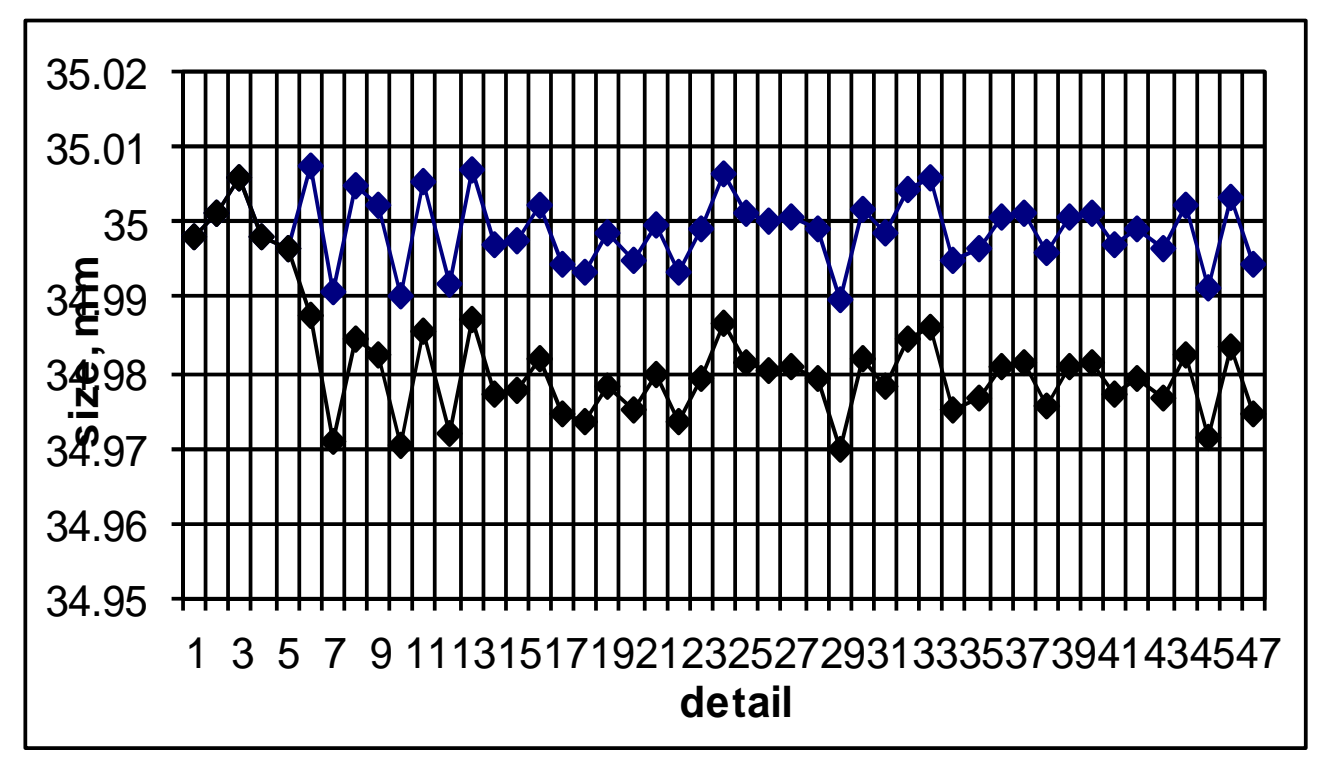

Fig.1. Diagrams of the process without dominant systematic factor

When the processing detail of the algorithm, the sample sizes comply with manufacture time during processing of the workpiece. As already mentioned, the minimum sample size is 3 details. When manufacture time is less and dimensional wear does not occur dynamically during the process, the average size is verified through a larder time interval (number details). It is possible be made for time interval in algorithm, during which the sequence of $n$ workpiece to be measured. So the principle of small current samples will be used. This will shorten the total time for the control of parts that are processed. 


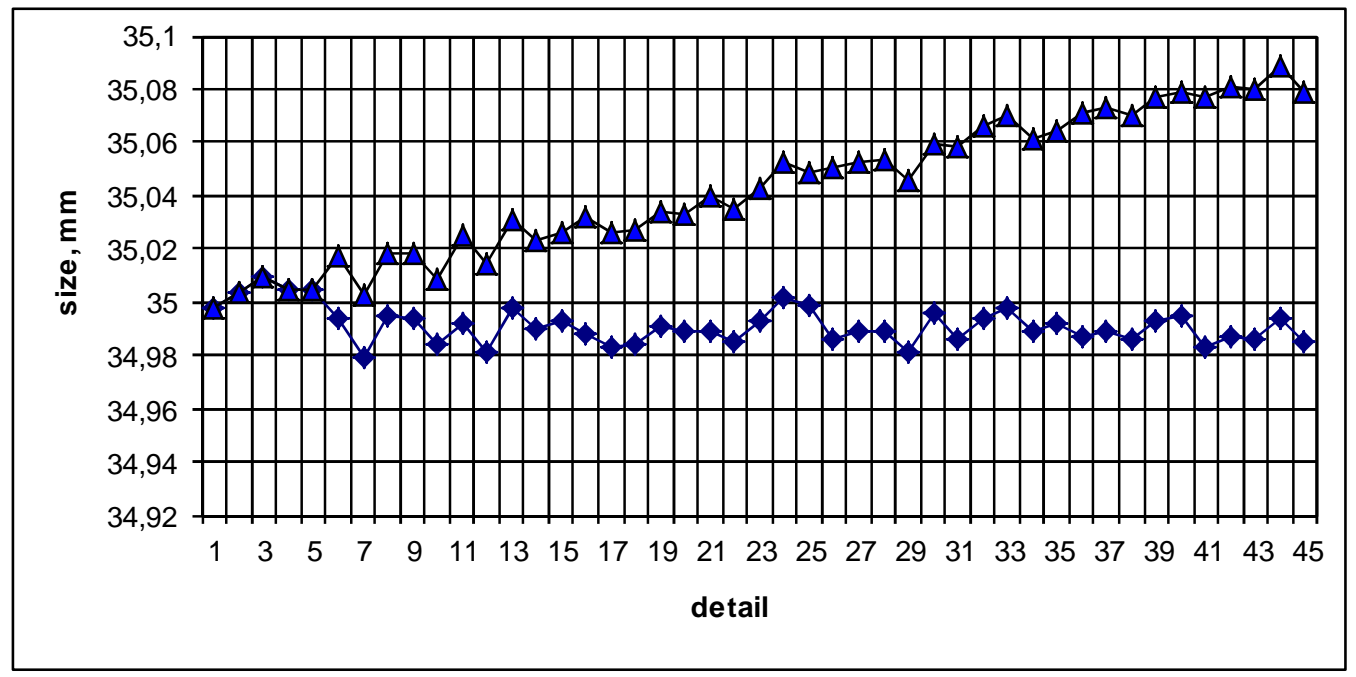

Fig. 2. Diagrams of the process with dominant systematic factor

\section{CONCLUSION}

Statistical process control (SPC) can help manufacturers improve the quality of their products. Proposed is a relatively simple and effective method and algorithm for computer control accuracy in turning CNC machine tools using the methods of statistical process control. Examined the possibilities of the proposed method by computer simulation of the process. It shows good performance as in processes without systematic dominant factor and expressed systematically varying the dimensions of tool wear. By using the proposed algorithm scattering of size is placed in the middle of the tolerance zone. It is optimal to abtaining of minimal probability of rejects, both the upper and the lower limit of the tolerance zone. Various variants carrying out the method are proposed adequate on the machining time during processing details in the sample. This can optimize the total time for processing of the batch.

\section{REFERENCES}

[1] W. Cochran, Sampling Techniques. Third ed. Wiley, 1977.

[2] M. Enchev and S. Koleva, Transitional process in initial dimensional setting with setup of details. X International
Congress "Machines, technologies, materials», September, 1820, Varna, Bulgaria, 2013.

[3] P. Hadjiski and C. Kaldashev, Programming of CNC machine tools with CAM systems. Technical University-Sofia, 2017.

[4] A..R Fazeli and E. Sharifi, Statistical Control and Investigation of Capability of Process and Machine in Wire Cut . EDM Process of Gas Turbine Blade Airfoil Tip. Engineering. 2011; 3: 260-265. http://www.SciRP.org/journal/eng _ [Accessed 2011].

[5] G.Falsone and D. Settineri, On the application of the probability transformation method for the analysis of discretized structures with uncertain proprieties. Probabilistic Engineering Mechanics, 35, 2014 pp.44-51, doi: 10.1016 /j. probengmech. 2013.10.001

[6] V.M. Nani, Statistical control of processing prismatic pieces on grinding machines, Measurement, 47, 2017, pp 516 - 520, (doi: 10.1016/ j.measurement. 2013.09.033

[7] B. Ramamoorthy, V. Radhakrishnan, A.Weckenmann, M. Knauer and D.A. Geus, Improvement of machining accuracy on a CNC lathe through error prediction and compensation. in: XV IMEKO World Congress, June 13-18, Osaka, Japan (1999)

[8] S. Kumar and S. T. Newman, Standardised Process Control System for CNC Manufacturing in: Advanced Design and Manufacturing Based on STEP, 2009, pp. 233-259.

[9] S. Salapatieva, Technology research for active control Turning CNC. PhD Thesis. TU-Sofia, branch Plovdiv, 2005. 